Schwannoma is a common soft tissue tumour, but it appears to be very rare in the gastrointestinal tract. Benign schwannoma develops extremely rarely in the mesocolon, with only 2 patients reported in the literature. A 75-year-old woman was admitted to our Department of Oncological Surgery with an abdominal mass, which was discovered incidentally during abdominal ultrasound examination. Positron emission tomography/ computed tomography imaging with the use of 18F-fluorodeoxyglucose (FDG $\mathrm{PET} / \mathrm{CT}$ ) showed an abnormal mass in the upper right abdomen with the presence of diffuse FDG uptake. A laparotomy revealed an encapsulated, non-invasive mesocolon tumour in the hepatic flexure region. Definitive diagnosis was confirmed by the histopathological examination of the postoperative preparation. Immunohistochemical staining confirmed benign mesocolon schwannoma. Previous cases indicate that schwannomas in the mesocolon are benign tumours. Our patient had a good prognosis even after enucleation treatment. Although schwannomas are very rare and generally asymptomatic, the differential diagnosis of schwannomas and gastrointestinal stromal tumours is important for practical purposes.

Key words: schwannoma, mesocolon, positron emission tomography, immunohistochemistry.

\section{Benign mesocolon schwannoma in PET/CT and immunohistochemistry} assessment: a case report

\author{
Zbigniew Kula ${ }^{1}$, Wojciech Jóźwicki ${ }^{2}$, Wojciech Zegarski ${ }^{3}$
}

${ }^{1}$ Department of Endoscopy, F. Łukaszczyk Oncology Center, Bydgoszcz, Poland 2Department of Tumor Pathology and Patomorphology, Ludwik Rydygier Collegium Medicum in Bydgoszcz, Nicolaus Copernicus University in Toruń, F. Łukaszczyk Oncology Center, Bydgoszcz, Poland

${ }^{3}$ Department of Oncological Surgery, Ludwik Rydygier Collegium Medicum in Bydgoszcz, Nicolaus Copernicus University in Toruń, F. Łukaszczyk Oncology Center, Bydgoszcz, Poland

\section{Introduction}

Schwannoma (neurilemmoma) is a common soft tissue tumour that occurs usually in the extremities, trunk, head and neck, mediastinum, retroperitoneum, and pelvis, but it appears to be very rare in the gastrointestinal tract [1]. Published reports mainly describe gastric schwannomas. These tumours occur uncommonly in the other segments of the alimentary tract from the oesophagus to the rectum [2-5]. Schwannomas develop extremely rarely in the pancreas [6, 7], mesentery [8, 9] and mesocolon [10, 11]. We report a case of an asymptomatic colon mesenteric schwannoma that was found incidentally.

\section{Case presentation}

The patient was a 75-year-old woman with an abdominal mass who was admitted to our Department of Oncological Surgery, The Lukaszczyk Oncology Center, Bydgoszcz, Poland. The patient did not have any gastrointestinal tract symptoms. The tumour was discovered incidentally in the abdominal ultrasound examination. It was a homogeneous hypoechogenic structure with a welldefined margin and was located medial to the right kidney, below the level of the head of the pancreas. A series of laboratory examinations, including tumour marker, showed normal results. Endoscopic examination did not show any abnormalities of the stomach, duodenum and colon. Positron emission tomography/computed tomography with the use of $18 \mathrm{~F}$-fluorodeoxyglucose (FDG PET/CT) imaging revealed the presence of an uneven lesion, $42 \mathrm{~mm} \times$ $\times 30 \mathrm{~mm} \times 52 \mathrm{~mm}$ in size, located at the level of the lower half of the right kidney, in front of the kidney, as well as an elevated glucose metabolism focused in this area, $34 \mathrm{~mm} \times 43 \mathrm{~mm} \times 37 \mathrm{~mm}$ in size (Fig. 1). The standardized uptake value (SUV) was 2.8. There was no evidence of invasion of the adjacent organs. Based on the patient's history, clinical findings, and PET/CT images the patient was referred for surgery. A laparotomy revealed an encapsulated, non-invasive mesocolon tumour in the hepatic flexure region. Simple enucleation of the tumour was performed without bowel resection. The tumour was well defined, encapsulated, $5.5 \mathrm{~cm}$ in diameter, with a smooth surface. The crosssection revealed a solid mass with grey and cherry colour. The pathology report showed the presence of spindle-shaped cells with nuclear palisading (Fig. 2A). No mitotic figures and no atypical appearance were observed. Immunohistochemically, the tumour cells were diffusely positive for S-100 protein (Fig. 2B) and vimentin (Fig. 2C) but negative for smooth muscle actin (SMA), CD 117, Ki67, desmin, CK MNF and CD 34 (Fig. 2D). The morphology and immunoprofile were characteristic for cellular schwannoma. The patient had a postoperative follow-up without any signs of recurrence during 24 months after the operation. 

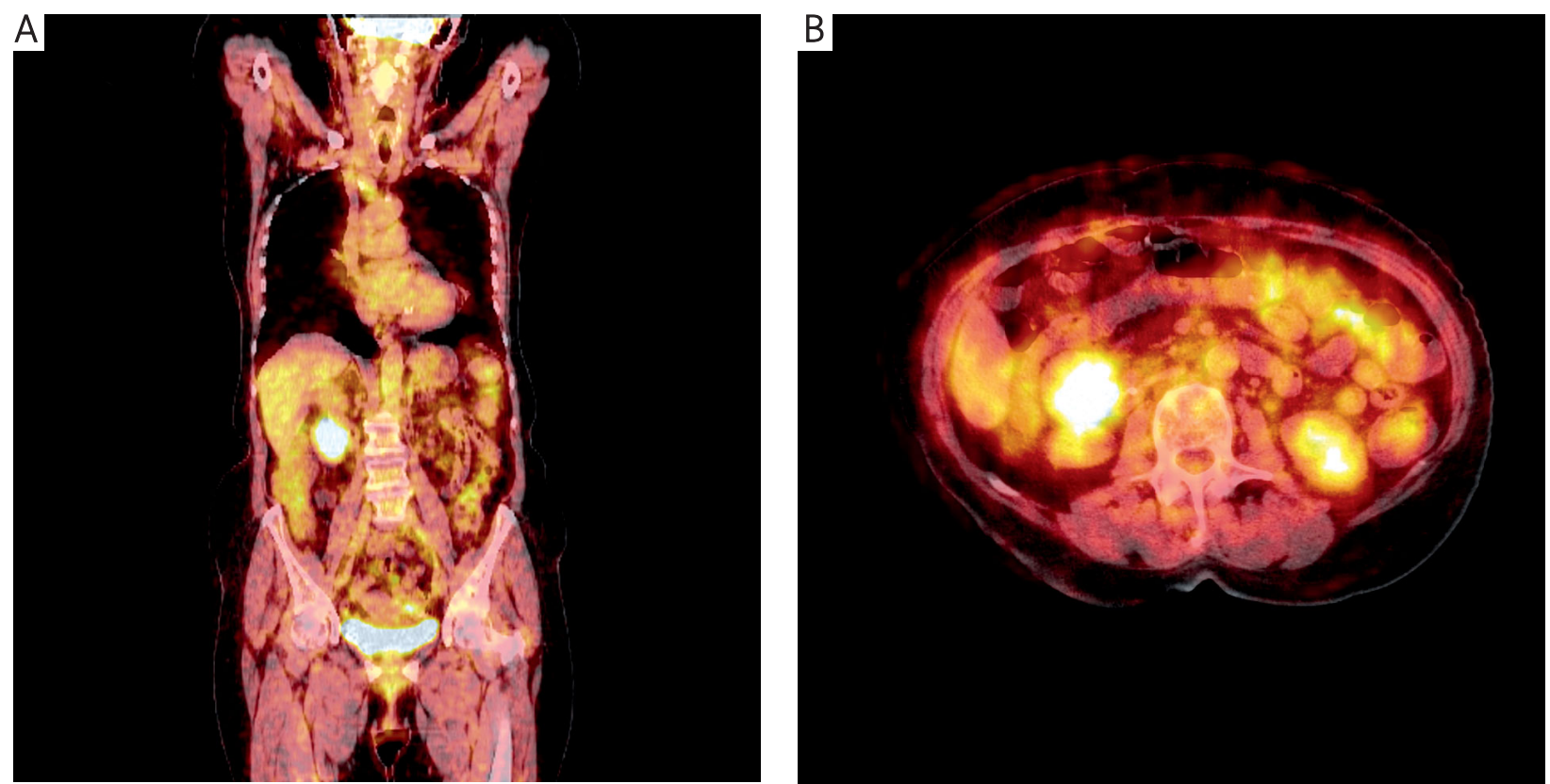

Fig. 1. Coronal (A) positron emission tomography/computed tomography with the use of 18F-fluorodeoxyglucose (FDG PET/CT) images of the trunk and transverse (B) FDG PET/CT images of the abdominal cavity showing abnormal mass. Focal FDG uptake in the tumour (Nuclear Medicine Department, The Oncology Center, Bydgoszcz)

FDG - 18F-fluorodeoxyglucose; PET/CT - positron emission tomography/computed tomography

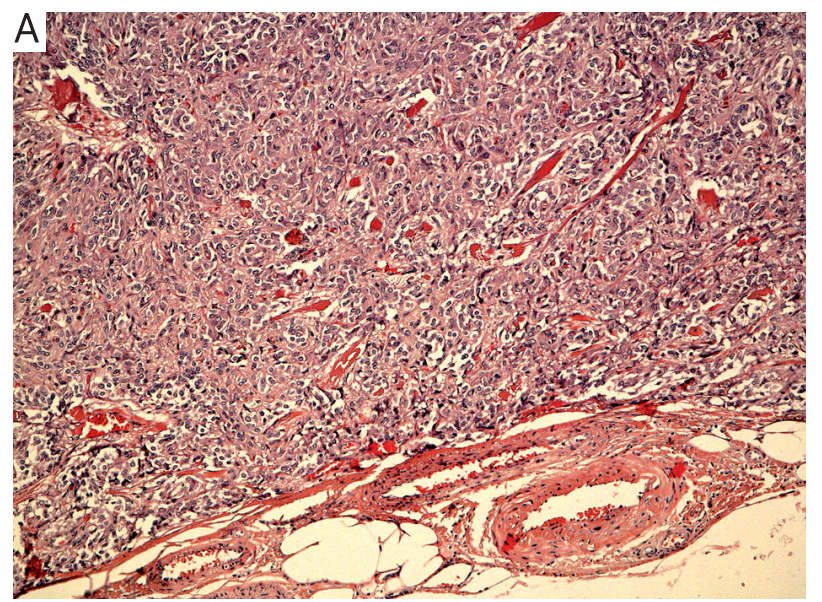

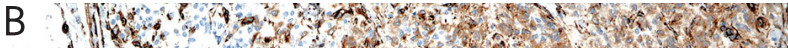

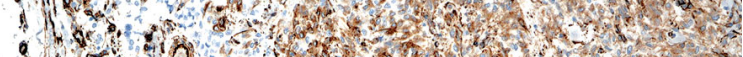

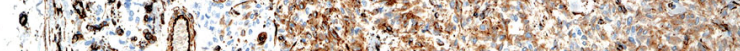

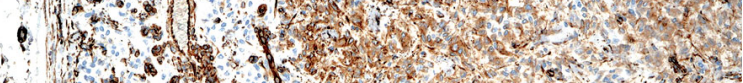

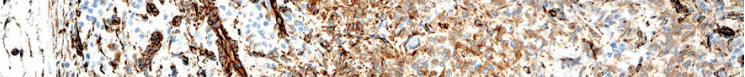

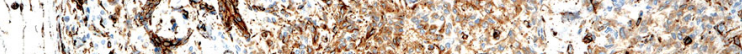

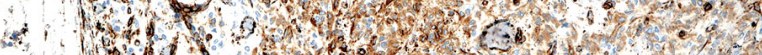

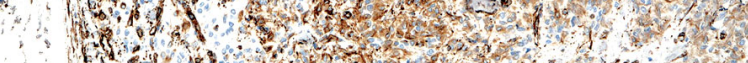
W.

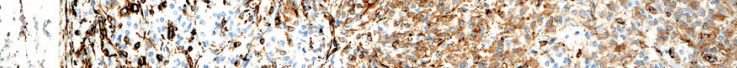

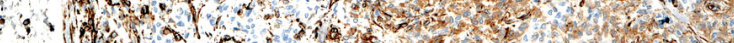

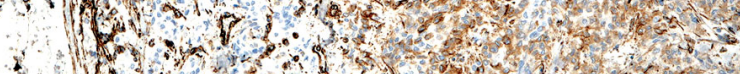

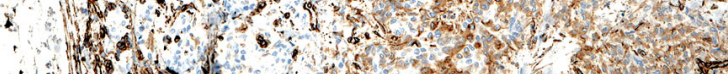

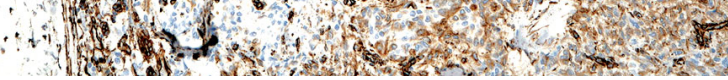
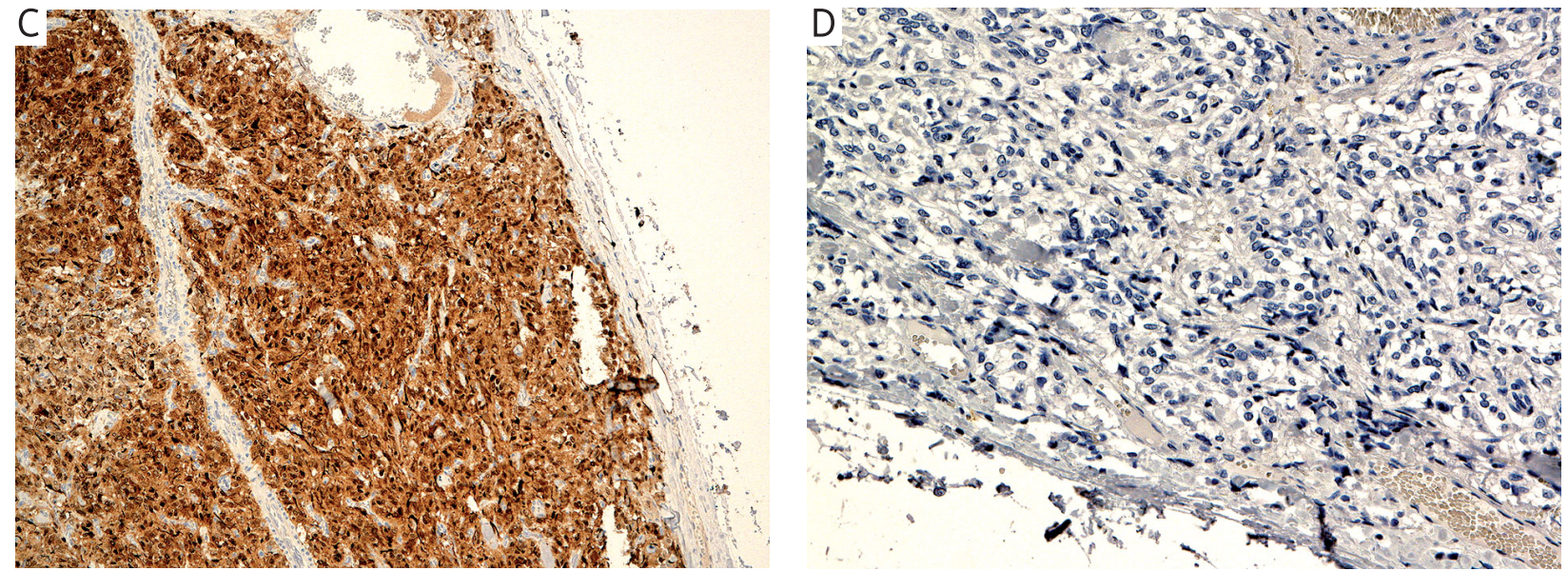

Fig. 2. Histological features of a schwannoma. This is a spindle-cell lesion (A), magnification 10x. The cells express S-100 protein (B) and vimentin (C), magnification 10x; are CD117 negative (D), magnification 20x 


\section{Discussion}

We report a case of an asymptomatic colon mesenteric schwannoma that was found incidentally. To the best of our knowledge, this is the third case of a schwannoma reported in the mesocolon. Schwannoma is a common soft tissue tumour, but appears to be rare among spindle-cell mesenchymal tumours of the gastrointestinal tract. Hou and coauthors [2] reported 33 benign schwannomas, which represented $2.9 \%$ of gastrointestinal mesenchymal tumours in that material. The symptoms of schwannomas in the gastrointestinal tract depend on the tumour location and size. Oesophageal schwannomas often occur simultaneously with dysphagia, whereas gastric schwannomas often occur with abdominal pain, discomfort in epigastria or bleeding. Colon or intestinal schwannomas can cause flatulence, constipation and alimentary tract obstruction symptoms. Schwannomas are slowly growing tumours that are usually present several years before the diagnosis. The asymptomatic course of the disease was confirmed in our patient. In most cases, only larger tumours in the advanced stage manifest alimentary tract obstruction symptoms caused by the external pressure. The diagnosis of mesocolon and mesenteric schwannoma as well as gastrointestinal intramural location is very difficult. CT and magnetic resonance imaging are most often used to diagnose and evaluate the stage of the tumour. In patients such as ours, FDG PET/CT can also be used to confirm the effectiveness of conventional methods in evaluating the tumour. We eliminated the presence of distant metastases based on FDG PET/CT examination, which also allowed us to evaluate the patient for surgery. The observed FDG uptake of the tumour suggested its malignancy. We suspected gastrointestinal stromal tumour (GIST) because of the elevated glucose metabolism, which is common in this type of tumour. FDG PET/CT imaging may have significant clinical value in the diagnosis of the postoperative recurrence of GIST after surgical treatment and monitoring the treatment response to tyrosine kinase inhibitor [12]. However, because FDG uptake is also characteristic for schwannomas, the SUV can indicate the malignancy of the tumour. Most gastrointestinal mesenchymal neoplasms are gastrointestinal stromal tumours (GISTs) or smooth muscle types. Schwannomas clinically resemble GISTs. The distinction between schwannoma and GIST is important because the former is benign even when large and mitotically active. Schwannomas are positive for S-100 protein and negative for desmin, actin and CD34 and CD117. Gastrointestinal schwannomas are easily distinguished from leiomyomas. Histologically, schwannomas usually show a spindle-cell pattern, similarly to cellular schwannomas with vague nuclear palisading, and often have sprinkled lymphocytes and a nodular lymphoid cuff [13]. In our case of a benign tumour a simple enucleation was a sufficient procedure, whereas malignant tumours would require standard oncological resection.

\section{Conclusions}

We report a case of an incidentally identified mesocolon schwannoma treated surgically. Despite the rarity, schwannoma must be considered as one of the possibilities in the differential diagnosis, as well as other mesenchymal tumours of the gastrointestinal tract, especially gastrointestinal stromal tumour. The definitive diagnosis can only be made on the basis of immunohistochemistry.

The authors thank Jaroslaw Jaroszek, MD, Alicja Weishof, MD and Bogdan Malkowski, MD for clinical and laboratory support of the case.

\section{References}

1. Weiss SW, Goldblum JR. Benign Tumours of Peripheral Nerves. Mosby, St. Louis 2001.

2. Hou YY, Tan YS, XU JF, Wang XN, Lu SH, Ji Y, Wang J, Zu XZ. Schwannoma of the gastrointestinal tract: a clinicopathological, immunohistochemical and ultrastructural study of 33 cases. Histopathology 2006; 48: 535-545.

3. Goh BKP, Chow PKH, Kesavan S, Yap W, Ong H, Song I, Eu K, Wong W. Intraabdominal schwannomas: a single institution experiene. J Gastrointestinal Surg 2008; 12: 756-60.

4. Sarlomo-Rikala M, Mittinen M: Gastric schwannoma - a clinicopathological analysis of six cases. Histopathology 1995; 27: 355-60.

5. Vinhais SN, Cabrera A, Nobre-Leitao C, Cunha TM. Schwannoma of the esophagus: computed tomography and endosonographic findings of a special type of schwannoma. Acta Radiol 2005; 45: 718-20.

6. Soumaoro LT, Teramoto K, Kawamura T, Nakamura N, Sanada T, Sugihara K, Arii S. Benign schwannoma of the pancreas. J Gastrointest Surg 2005; 9: 288-90.

7. Wu YL, Yan HC, Chen LR, Chen J, Gao SL, Li JT. Pancreatic benign schwannoma treated by simple enucleation: case repost and review of literature. Pancreas 2005; 31: 286-8.

8. Minami S, Okada K, Matsua M, Hayashi T, Kanematsu T. Benign mesenteric schwannoma. Gastrointestinal Images 2005; 9: 1006-8.

9. Khan AA, Schizas AM, Cresswell AB, Khan MK, Khawaja HT. Digestive tract schwannoma. Dig Surg 2006; 23: 265-9.

10. Quandalle P, Belbenoit C, May JP, Houcke, Ribet M: Schwannome malin du meso-sigmoide. Lille Medical 1966; 11: 577-80.

11. Angamuthu N, Rozario A. Schwannoma of the transverse mesocolon. Indian J Gastroenterol 2003; 22: 198-9.

12. Goerres GW, Stupp R, Barghouth G, Hany TF, Pestalozzi B, Dizendorf E, Luthi F, von Schulthess GK, Leyvraz S. The value of PET, CT and in-line PET/CT in patients with gastrointestinal stromal tumours: long-term outcome of treatment with imatinib mesylate. Eur J Nucl Med Mol Imaging 2005; 32: 153-62.

13. Miettinen M, Blay JY, Sobin LH. Mesenchymal tumours of the stomach. In: Pathology and Genetics of Tumour of the Digestive System. Hamilton SR, Aaltonen LA (eds). IARCPress, Lyon 2000; 62-65.

\section{Address for correspondence}

\section{Zbigniew Kula}

Department of Endoscopy

Oncology Centre - Prof. Lukaszczyk Memorial Hospital

dr I. Romanowskiej 2

85-796 Bydgoszcz

e-mail: zbigniew.kula@co.bydgoszcz.pl

Submitted: $\quad 10.05 .2011$

Accepted: $\quad 18.01 .2012$ 